\title{
Study of the Leutwyler-Smilga regimes: Lessons for full QCD simulations*
}

\author{
S. Dürr ${ }^{\text {a }}$

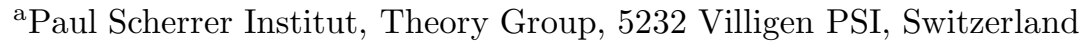

Some key points out of my recent study of the characteristic features of the small $(x \ll 1)$, intermediate $(x \simeq 1)$ and large $(x \gg 1)$ Leutwyler-Smilga regimes for QCD-type theories in a finite volume $(x=V \Sigma m)$ are presented, and a few immediate consequences for full QCD simulations are discussed.

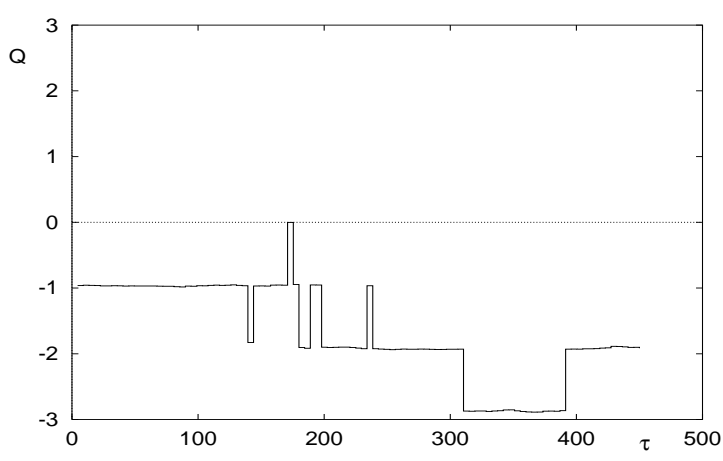

Figure 1. Time history of $\nu_{\text {nai }}$ in a HMC simulation with a quadruple of staggered quarks at $\beta=5.35, m=0.01$ (figure taken from no. 3 in $[\mathbb{1}]$ ).

\section{Motivation}

Numerical simulations of full QCD may be hampered by the fact that certain observables show rather long autocorrelation times. A well known example is the global topological charge $\nu$ which was found to develop problems for sufficiently small quark masses: For staggered fermions an insufficient tunneling rate has been observed both in the symmetric and in the broken phase [1]. For Wilson type sea-quarks mobility between the topological sectors was found to be satisfying for $M_{\pi} / M_{\rho}>0.56$, but the same problem emerges if $\kappa$ is tuned sufficiently close to $\kappa_{\text {crit }}$ [2]. Fig. 1 displays the time-history of a simulation representing the "worst case scenario"; the sample is obviously non-ergodic, since the generated $\nu$-distribution is far from symmetric.

Simply prolonging the simulation time may not

\footnotetext{
${ }^{*}$ Talk given at LAT'00, Bangalore, 17 - 22 Aug 2000.

${ }^{\dagger}$ E-mail: stephan.duerr@psi.ch
}

be an option; hence one is left with the question whether the given degree of (non-)ergodicity is tolerable for practical purposes or whether an ensemble average on the basis of the data available is afflicted with a sizable systematic error. It is clear that the answer may depend on the observable one has in mind. Hence from a physical point of view the question may be rephrased as follows: Does a given observable on a perfect (full) QCD ensemble - generated in a box of given volume $V$ and with $N_{f}$ sea-quarks of mass $m$ - depend on the topological charge $\nu$ of the background?

\section{Leutwyler-Smilga analysis in a nutshell}

For pionic observables, much can be said on purely analytical grounds. Leutwyler and Smilga (LS) have shown [3] that a clear answer is possible for two regimes of quark masses and box volumes, both of which involve the LS-parameter

$x \equiv V \Sigma m$,

which indicates whether the box is "small" or "large" in the sense that it decides on whether the systems prefers to show symmetry restoration or SSB phenomena, respectively: For $x \ll 1$ chiral symmetry is effectively restored and quarks and gluons represent appropriate degrees of freedom. For $x \gg 1$ the $S U\left(N_{f}\right)_{A}$ symmetry is quasibroken, meaning that - though the box-volume is formally finite - pions represent appropriate degrees of freedom $\left(N_{f} \geq 2\right)$. Note that $m$ refers to the mass of the sea-quarks and that $x$ is (in principle) independent of the parameter $M_{\pi} L$.

$\overline{{ }^{1} \Sigma=-\lim _{m \rightarrow 0} \lim _{V \rightarrow \infty}}\langle\bar{\psi} \psi\rangle$ (this order) is the condensate in the chiral limit; both $\Sigma$ and $m$ are scheme- and scale-dependent, but the combination is an RG-invariant. 

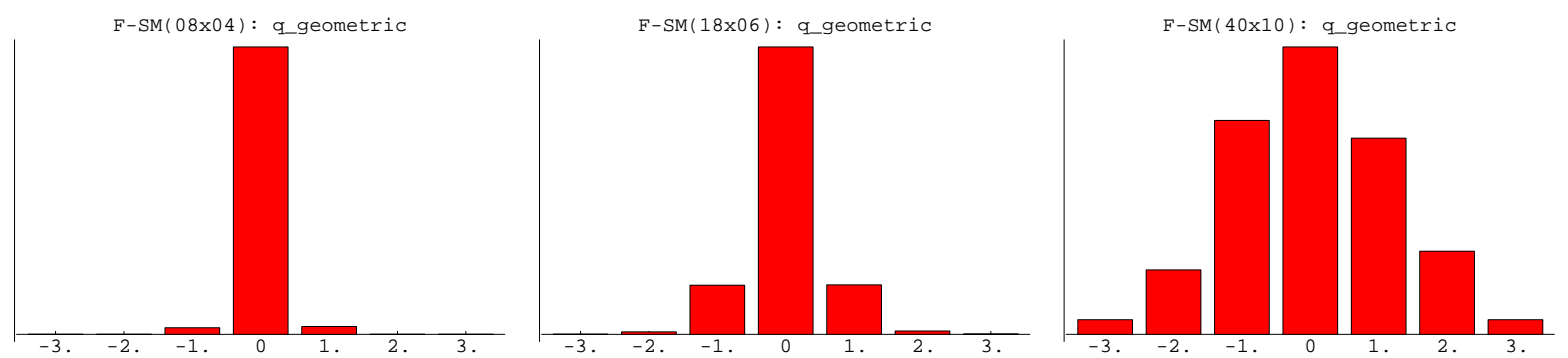

Figure 2. Distribution of $\nu_{\text {geo }}$ in the small (8x4 lattice), intermediate (18x6) and large (40x10) LeutwylerSmilga regimes, respectively $\left[\mathrm{QED}(2)\right.$ data for $\left.\beta=3.4, m=0.09, N_{f}=2\right]$.

The net result of the LS-analysis [3] is that for $x \ll 1$ the partition function is completely dominated by the contribution from the topologically trivial sector for small enough $m$, since

$Z_{\nu} \propto m^{N_{f}|\nu|}$.

In the opposite regime $x \gg 1$ and with the additional condition that the quark masses are so light that the sea-pion overlaps the box $\left(M_{\pi} L \ll 1\right)$, the path-integral in the effective description (requires $\Lambda_{\text {eff }} L \gg 1$ ) is dominated by the constant mode (4], and Leutwyler and Smilga end up finding that the partition function is approximately independent of $\nu$ or, more precisely, that the distribution is [3]

$Z_{\nu} \propto e^{-\frac{\nu^{2}}{2\left\langle\nu^{2}\right\rangle}}$ with $\left\langle\nu^{2}\right\rangle=\frac{V \Sigma m}{N_{f}}$.

\section{Checking the net charge distribution}

Since the LS-issue is peculiar to the full theory, a preliminary study in a QCD-type theory seems permissible. The massive multiflavour Schwinger model (QED(2) with $N_{f} \geq 2$ ) is supposed to reproduce all qualitative features [5]. I use the Wilson gauge action $S_{\text {gauge }}=\beta \sum\left(1-\cos \theta_{\square}\right)$ and a pair of (dynamical) staggered fermions.

I compare the three regimes $x \ll 1, x \simeq 1, x \gg 1$ to each other using three dedicated simulations: Working with fixed staggered mass $m=0.09$ and $\beta=1 / g^{2}=3.4$, the three regimes are represented by the three volumes $V=8 \times 4,18 \times 6,40 \times 10$. From this setup the LS-parameter takes the values $x \simeq 0.33,1.12,4.16$, respectively, and the pion (pseudo-scalar iso-triplet) has a (common) mass $M_{\pi}=0.329$ and therefore a correlation length $\xi_{\pi}=3.04$ as to fit into the box.
A configuration is assigned an index only if the geometric $\left(\nu_{\text {geo }}=\frac{1}{2 \pi} \sum \log U_{\square}\right)$ and the fieldtheoretic definition $\left(\nu_{\mathrm{fth}}=\kappa \nu_{\text {nai }}, \nu_{\text {nai }}=\sum \sin \theta_{\square}\right.$, $\kappa \simeq 1 /\left(1-\left\langle S_{\text {gauge }}\right\rangle / \beta V\right)$, after rounding to the nearest integer, agree - for details see [5].

As one can see from Fig. 2, the charge distributions found in the three runs seem to follow a general pattern consistent with the LS-prediction: For $x \ll 1$ the distribution (and therefore the partition function) is dominated by the contribution from the topologically trivial sector, whereas for $x \gg 1$ the distribution gets broad and seems compatible with the gaussian form (3) with variance $x / N_{f}$, i.e. $\sigma^{2}=\left\langle\nu^{2}\right\rangle=x / 2$.

\section{Role of the functional determinant}

Since the LS-issue is peculiar to the full (unquenched) theory, an attempt to understand by which mechanism the three regimes differ from each other may lead one to investigate how the functional determinant or specifically its contribution to the total action per continuum-flavour

$S_{\text {fermion }}=-\log (\operatorname{det}(\not D+m))+$ const

relates to the contribution from the gauge field and, in addition, how this might depend on the topological charge of the background. The idea is thus to study such a relationship sectorally, i.e. after the complete sample has been separated into subsamples with definite absolute charge $|\nu|$.

From the scatter plots in Fig. 3 one notices a (weak) positive correlation between $S_{\text {fermion }}$ and

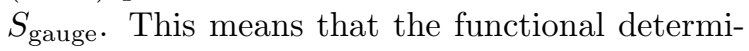
nant acts - roughly - like an effective renormalization of $\beta$ with a factor bigger than 1 . A key observation is that the correlation improves, if one 

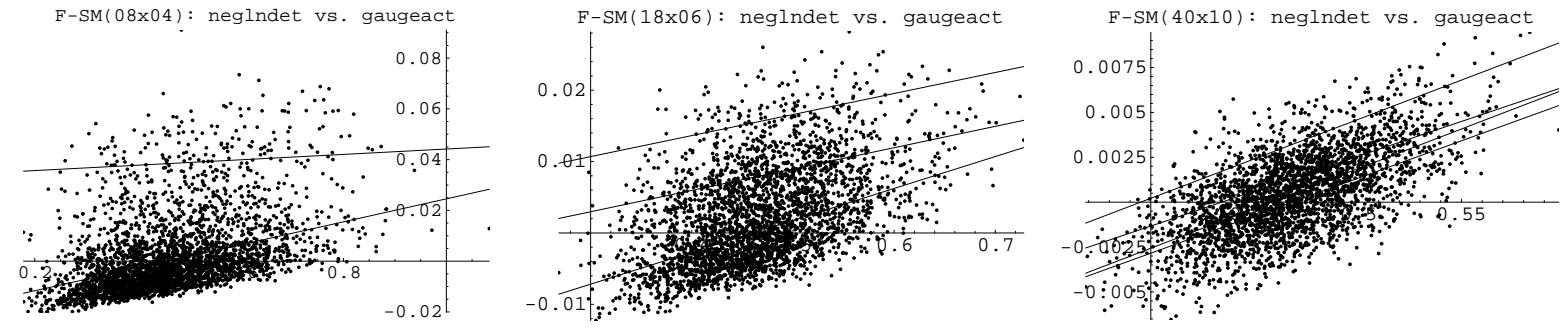

Figure 3. $\quad S_{\text {fermion }}=-\log \left(\operatorname{det}(\not D+m)\right.$ ) (i.e. per continuum flavour) versus $S_{\text {gauge }}$ together with the best linear fits for the reasonably populated topological sectors in the small (8x4 lattice), intermediate $(18 \mathrm{x} 6)$ and large (40x10) Leutwyler-Smilga regimes, respectively $\left[\mathrm{QED}(2)\right.$ data for $\left.\beta=3.4, m=0.09, N_{f}=2\right]$.

separates the sample into subsamples with fixed $|\nu|$ - for each one of which the best linear fit for $S_{\text {fermion }}$ as a function of $S_{\text {gauge }}$ is included. Quite generally, the linear function for low $|\nu|$ lies below the one(s) for higher $|\nu|$, and has a larger slope than the latter. This means that the functional determinant brings - in general - an overall suppression of higher topological sectors w.r.t. lower ones and a sectorally different renormalization of $\beta$. There are interesting effects as $x$ varies: In the small LS-regime both the sectoral dependence of the renormalization factor and the offset between neighboring sectors are huge, i.e. for $x \rightarrow 0$ the functional determinant acts as a constraint to the topologically trivial sector. For $x \simeq 1$ both offset and sectoral dependence of the slope are smaller. In the large LS-regime there is only a minor overall suppression of higher topological sectors w.r.t lower ones, and the effective renormalization of $\beta$ (and hence, in 4 dimensions, of the physical lattice spacing) seems to be uniform for all sectors.

\section{Sectoral heavy-quark potential}

It is instructive to play the same game with a standard observable like the heavy-quark potential. As one can see from the leftmost column in Fig. 4, the physical potential (on the unseparated sample) shows, by its curvature, clear evidence of the dynamical fermions - the quenched potential would be strictly linear. The remaining columns show for each LS-regime the sectoral heavy-quark potentials on the subsets with lowest and highest (reasonably populated) $|\nu|$. The sectoral dependence is found to be striking in the regime $x \ll 1$, where the two subpotentials are clearly inconsistent with each other. For intermediate $x$, there is still a clear disagreement between the two extreme- $|\nu|$ subpotentials shown. In the regime $x \gg 1$, finally, data show good consistency between neighboring topological sectors (e.g. $\nu=0$ and $\nu= \pm 1-$ see [月]), but there is no clear evidence of an overall consistency. In fact, the sectoral potentials for $\nu=0$ and $\nu= \pm 3$ seem to indicate that even in the large LS-regime a remnant sectoral dependence might persists upon generating extreme changes in the absolute value of the topological charge. Of course it is possible that in the large- $x$ simulation at hand the value of $x$ (about 4.16) is not yet large enough for the large- $x$ behaviour to be fully pronounced, but the rule of thumb seems to be that in the large- $x$ regime standard physical observables may be sensitive to distortions of the $\nu$-histogram which are large compare to its natural width $\sigma \simeq\left(x / N_{f}\right)^{1 / 2}$.

\section{Lessons for full QCD simulations}

Each one of the LS-regimes has its characteristic features: For $x \ll 1$ correct sampling w.r.t. $\nu$ is easy to achieve; a simple constraint to the topologically trivial sector proves useful. For $x \simeq 1$ the situation is much more delicate, since this is the regime where good sampling w.r.t. the topological charge is both crucial and nontrivial to achieve. For $x \gg 1$ standard observables (i.e. unrelated to the $U(1)_{A}$-issue) prove fairly insensitive on the $\nu$ distribution - unless the latter suffers from extreme distortions, i.e. on a scale which is large compared to its natural width $\sigma \simeq\left(x / N_{f}\right)^{1 / 2}$.

In spite of the good news that state-of-the-art simulations of full QCD operate in the large- $x$ 

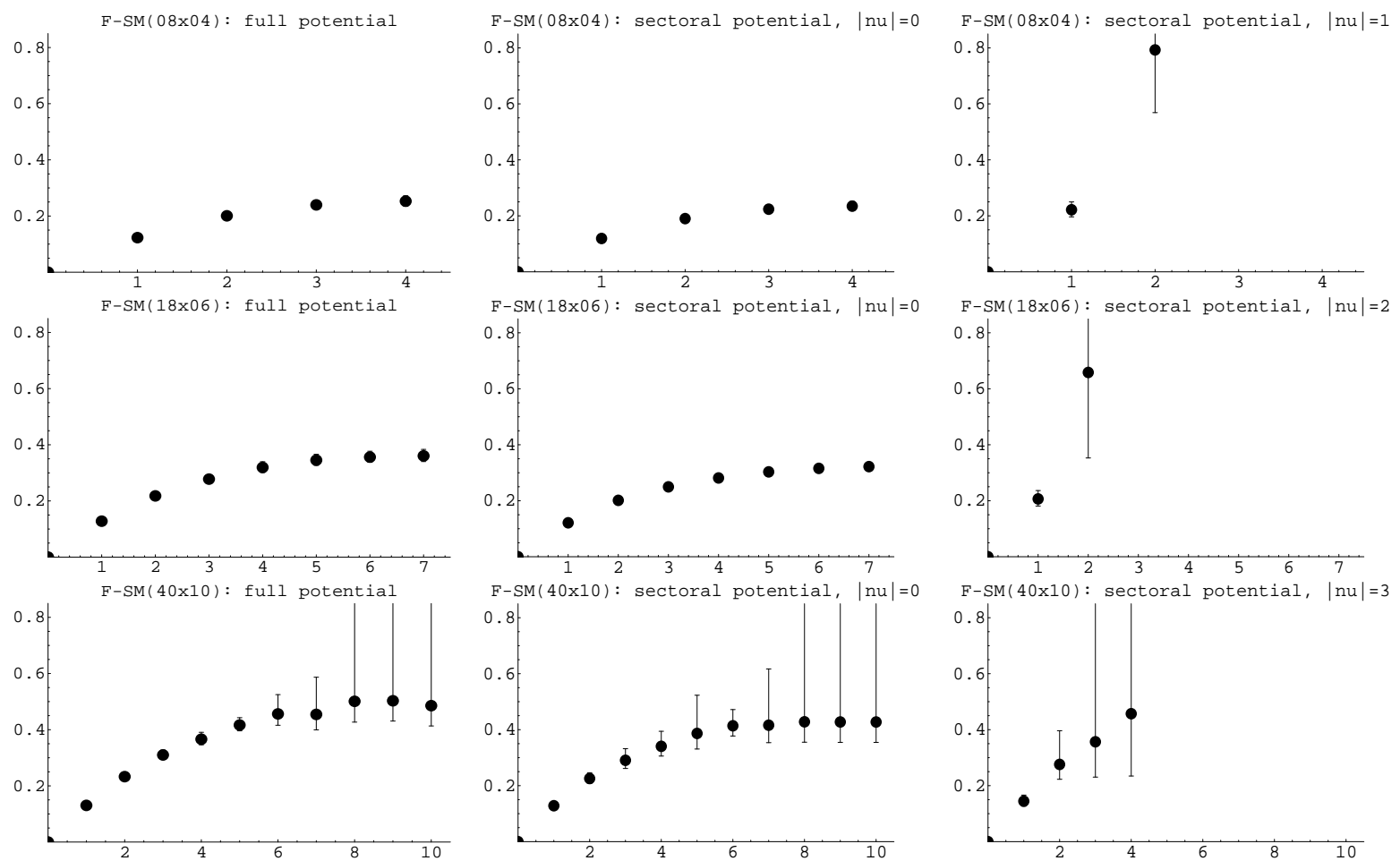

Figure 4. Physical heavy-quark potential (leftmost column) and selected sectoral versions in the small $(V=8 \times 4)$, intermediate $(18 \times 6)$ and large $(40 \mathrm{x} 10)$ Leutwyler-Smilga regimes, respectively $[\mathrm{QED}(2)$ data].

regime [6], there is - in principle - a problem with chiral extrapolations. It derives from the fact that the key role of the LS-parameter is to determine which one of the limits $m \rightarrow 0$ and $V \rightarrow \infty$ is in the "inner" position and thus winning: $x \gg 1$ corresponds to $\lim _{m \rightarrow 0} \lim _{V \rightarrow \infty}$, whereas $x \ll 1$ implements a situation like $\lim _{V \rightarrow \infty} \lim _{m \rightarrow 0}$. Therefore one likes to extrapolate from data-points won from a cascade of simulations for which $x$ increases (or stays at least constant and $\gg 1$ ) as the dynamical quark mass decreases. Looking at the definition (11), one realizes that $x$ decreases if a series of simulations on a given grid is used, i.e. one is marching in the wrong direction: If $\beta$ gets reduced for smaller $m$ so as to keep the lattice spacing constant (cf. Fig. 3 and the subsequent discussion), $x$ decreases only in proportion to $m$; if one works at fixed $\beta$, the situation is of course worse. To correct, one would have to use larger grids for smaller quark masses. While in current state-of-the-art simulations the box to start with seems sufficiently large (or the quark mass to end up with heavy enough) as to prevent this effect from getting numerically important [6], it is clear that this is a point one may wish to control as the quest for lighter quark masses goes on.

\section{REFERENCES}

1. Y. Kuramashi et al., Phys. Lett. B 313, 425 (1993); M. Müller-Preussker, Proc. XXVI Int. Conf. on High Energy Physics, Dallas, TX, 1992, ed. J.R. Sanford, AIP Conf. Proc. No. 272, 1545 (1993); B. Allés et al., Phys. Lett. B 389, 107 (1996).

2. B. Allés et al., Phys. Rev. D 58, 071503 (1998).

3. H. Leutwyler and A.V. Smilga, Phys. Rev. D 46, 5607 (1992).

4. J. Gasser and H. Leutwyler, Phys. Lett. B 188, 477 (1987).

5. S. Dürr, hep-lat/0008022.

6. Result of a private discussion with R. Burkhalter on the basis of actual CP-PACS data. 